\title{
Afinando la Estrategia: los enfoques de la Gestión Administrativa y la orientación del Mercado
}

\author{
Refining Strategy: Management approaches and Market orientation
}

\author{
Ricardo Verján-Quiñones* \\ Universidad Autónoma de Baja California - México \\ ORCID iD: https://orcid.org/0000-0001-6624-0873 \\ ricardoverjan@uabc.edu.mx
}

\author{
María G. Woo-Gutiérrez \\ Universidad Autónoma de Baja California - México \\ ORCID iD: https://orcid.org/0000-0002-6470-6788 \\ guadalupe.woo@uabc.edu.mx
}

\author{
Daniel García-Alegre \\ Universidad Autónoma de Baja California - México \\ ORCID iD: https://orcid.org/0000-0003-3054-030X \\ daniel.garcia77@uabc.edu.mx
}

* Autor a quien debe ser dirigida la correspondencia
Fecha de recepción: 28/09/2021

Fecha de evaluación: 05/10/2021

Fecha de aceptación: 01/11/2021

Cómo citar: Verján-Quiñones, R., Woo-Gutiérrez, M., \& Garcia-Alegre, D. (2021). Afinando la Estrategia: los enfoques de la Gestión Administrativa y la orientación del Mercado. Revista Cientifica Anfibios, 4(2), 29-35. https://doi.org/10.37979/afb.2021v4n2.92

\section{Resumen}

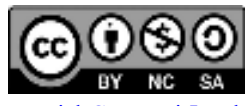

Atribución-NoComercial-CompartirIgual 4.0 Internacional

Llevar una adecuada gestión de marketing en las empresas implica ir implementando ajustes sobre la marcha que permitan el desarrollando estrategias que permitan posicionar a una marca, empresa, o producto. En el artículo a analizar se presenta dos aspectos importantes en el actuar de una industria en el mercado; por un lado, la gestión estratégica y por otro el marketing, pero sin duda ambas son fundamentales para que las organizaciones lleguen a alcanzar sus objetivos y metas propuestos. Es por esto que se plantea una revisión de la literatura que permita dilucidar los enfoques de la gestión administrativa y la orientación del mercado, con la finalidad que los resultados obtenidos se postulen como referencias para gerentes y organizaciones. Los resultados demuestran que el enfoque administrativo depende en gran medida del entorno económico de la organización y la orientación al cliente suele ser la más exitosa.

\section{Palabras clave}

Gestión estratégica; enfoque estratégico; orientación al mercado; orientación al cliente

\begin{abstract}
Adequate marketing management in companies serves to implement on the fly tools that allow the development of strategies to position a brand, company or product. In the article to be analyzed, two important aspects are presented in the performance of an industry in the market; on the one hand, strategic management and on the other hand, marketing, but undoubtedly both are essential for organizations to achieve their objectives and goals. For this reason, a review of the literature is proposed to elucidate the approaches to administrative management and market orientation, so that the results obtained can be used as references for managers and organizations. The results show that the administrative approach depends to a great extent on the economic environment of the organization and the customer orientation tends to be the most successful.
\end{abstract}

Keywords

Strategic management; strategic focus; market orientation; customer orientation 


\section{Introducción}

En los inicios de los años cincuenta y a lo largo de varias décadas, se ha afirmado que el concepto de marketing que sea cobijado bajo los brazos de las empresas que estén dispuestas a implementarlo como técnica y estrategia de crecimiento tendrán mejores resultados (Morgan \& Rego, 2009; Urde, 1994)

En el año 1988 el Marketing Science Institute, reconocido ponente de las investigaciones en el área de marketing, estableció los posibles efectos derivados de los estudios orientados a las empresas, clientes y sus mercados. Aplicando y reforzando lo antes dicho, a lo largo de la existencia del marketing, se ha dado a conocer las ventajas y beneficios que atribuyen el implementar una correcta orientación en el desarrollo de la mercadotécnica dentro de las empresas. Según Denison y McDonald (1995): "El éxito en marketing no consiste solamente en identificar cuál es la estrategia adecuada, sino, además, en poder implantarla correctamente"

Por otro lado, citando la misma conceptualización y filosofía de los autores antes mencionados, Lambin (1995) explica el hecho que: "Comprender el concepto marketing es una cosa, ponerlo en práctica es otra" siendo ésta en donde las empresas parecen haber hallado un mayor problema al limitarse a poner en práctica políticas de mezcla de marketing dirigidas a dominar el mercado a través de acciones de venta, dejando de lado las acciones estratégicas de segmentación de público objetivo y proposición de valor. Dicho mejor en otras palabras, se genera un carente marketing táctico de orientación al mercado alejándose del estratégico.

Es así que se plantea una investigación con el objetivo de ofrecer una herramienta para que las organizaciones afinen su estrategia respecto a los enfoques de la gestión administrativa y la orientación del mercado. Para tales fines se anuncian los referentes teóricos trayendo a colación los conceptos mas importantes asociados con estas temáticas en estudio y seguidamente se declaran los aspectos metodológicos tomando como base una revisión de la literatura de gestión que posibilite encontrar diferencias y semejanzas entre las temáticas propuestas y a partir de esta revisión plantear resultados y conclusiones respaldadas por evidencia teórica y empírica.

\section{Referentes Teóricos.}

Lloyd C. Harrys (2002) menciona que, al explorar el tema de las diferencias en los enfoques de gestión referentes a la orientación al mercado, se encuentra con estudios realizados a inicios de los años 90s en los que descuida la orientación al mercado.

En ese mismo sentido, dicho autor menciona que, en el estudio que realiza en 2002, en doce estudios de casos de diferentes empresas de servicios incluyendo cadenas de restaurantes, hoteles, empresas minoristas, empresas de servicios industriales y varios proveedores de servicios financieros. Mediante una muestra aleatoria en la que se selecciona a 500 empresas que en los últimos seis meses habían emprendido un esfuerzo de cambio orientado al mercado.

En dicho estudio se involucran más de 260 entrevistas aplicadas inicialmente a personal de primera línea o informante clave y posteriormente el investigador principal, identificaba a las personas cuya percepción de cambio a la orientación del mercado era valiosa.

Los resultados indican que los enfoques de gestión para desarrollar la orientación al mercado difieren en cinco dimensiones principales y cada empresa tiende a enfatizar al menos uno de estos énfasis.

Con el objetivo de abordar la brecha en el conocimiento existente a través del conocimiento empírico sobre las diferencias en la forma en que las gerencias intentan desarrollar una mejor orientación al mercado y proporcionar conocimientos con las variaciones en las estrategias, tácticas, métodos y énfasis de enfoques de gestión para desarrollar la orientación al mercado.

En cuanto a las barreras a la orientación al mercado, se menciona que existen dos tipos de barreras que consistentemente emergen como impedimentos; (1) las acciones, actitudes y comportamientos de los gerentes (2) las características organizacionales de la empresa, que son los factores que requieren mayor consideración.

Felton (1959) es el primero que realiza un análisis que destaca las actitudes, habilidades y comportamientos ejecutivos como barreras a la orientación del mercado y afirma que debido a 
las pocas capacidades con las que cuentan los ejecutivos se impide la orientación al mercado. Del mismo modo, estudios contemporáneos haces énfasis en que los defectos en el desarrollo son directamente atribuibles a a las limitaciones de la alta dirección (Jawaroski y Kholi1993; Harris y Piercy 1999; Van Egeren y col. 1999).

Por otro lado, los enfoques para desarrollar la orientación al mercado sugieren que las empresas deberían centrarse en la creatividad, la serendipia, el espíritu empresarial, la experimentación, la protección caníbal y los equipos competitivos (Kumar y col. 2002).

Existen teorías que pueden ayudar al desarrollo de la orientación al mercado. Uno de los más interesantes es el marketing interno, que propone el uso de técnicas de marketing en los miembros de la organización para promover valor y motivar a 1 personal de servicio y que al mismo tiempo promueve la visión de que los clientes internos y externos valen la pena y por lo tanto se afirma que es posible una mejor orientación al mercado (Lings, 1999).

Si bien el endomarketing o marketing interno es una estrategia que tiene como objetivo fortalecer la cultura organizacional de los clientes internos o colaboradores, sin importar el tamaño de la organización, es importante resaltar la importancia que estos tienen como contribución para el éxito de la empresa.

El énfasis de "corazones y mentes" durante el cambio orientado al mercado se manifiesta en un enfoque en la necesidad fundamentalmente para cambiar las actitudes y creencias existentes de los empleados hacia esos mercados, que se relaciona a su vez con el autocontrol y el foco no está en el cambio de comportamientos, sino más bien, en la alineación de actitudes de los empleados en todos los niveles, aunque principalmente adoptados por la dirección y que se pueden ver reforzados de forma positiva mediante recompensas financieras o de otra índole(Chen \& Chen, 2009; Larke et al., 2018; Madden et al., 2006; Park et al., 2010; Rojas De Francisco et al., 2016).

Para comenzar este proceso de cambio (Harris, 2002), menciona que para cambiar la filosofía de toda una corporación requiere no solo una profunda comprensión de la organización, sino también una visión objetiva y sin emociones que solo se puede obtener de personas ajenas que no se involucran, pero saben cómo hacerlo, y esto se logra mediante la contratación de agencias especializadas externas (Kashmiri et al., 2017; Morgan \& Rego, 2009; Nowlin et al., 2018; Piercy et al., 1997; Ramanathan et al., 2016).

Harris hace una aportación muy valiosa en la que menciona que seguidos de la instrucción de individuos e grupos en los principios de la orientación al mercado en el que se enfoque en el uso de programas para comunicar y enseñar lo que son concebidos para hacer habilidades, conocimientos y estructuras necesarias para un valor superior y en segundo lugar a centrarse en el retorno de mercado en el que la experiencia de aprendizaje adquirida a través de la interacción con el mercado, conduce a una mejora del mercado ya que se basa en la experiencia real (Frías-Navarro \& Pascual, 2012; Guzmán Duque \& Abreo Villamizar, 2017; Longbottom et al., 2000; Mueller \& Thomas, 2001; Nath \& Mahajan, 2008).

Es importante resaltar la importancia de la aplicación de las estrategias de servicio postventa en cualquier organización ya que, a largo plazo se crea una cultura de excelencia de servicio orientada al mercado que genera muchos beneficios, y es la forma más eficaz para desarrollar la orientación al mercado, se asegura de crear una buena experiencia que abona al retorno de los clientes y además se adquiere experiencia para la mejora continua de los procesos y técnicas internas (Aaker, 2008; Farzad; et al., 2008; Grinstein, 2008; Sainidis et al., 2019).

En este mismo sentido, se considera que el servicio de calidad es una ventaja competitiva inimitable y que, cuando es bien gestionada marca una diferencia significativa que se traduce en cambios en las personas que integran la organización y a su vez, es los clientes reales y potenciales (Fraj et al., 2015; Garcia et al., 2013; Guzmán Duque \& Abreo Villamizar, 2017; Kowalkowski et al., 2017; Lupoli Junior \& Dos Santos, 2019; Nowlin et al., 2018).

La clave para el desarrollo y mejora de la orientación al mercado es mediante el benchmarking, que consiste en evaluar y analizar los procesos, técnicas, productos o servicios, entre otros aspectos de otras compañías, con el objetivo de tomarlos como referencia para las estrategias futuras, esto con la intención de aprender de la ex- 
periencia de los demás y para mejora propia del desempeño de la organización (Augusto \& Coelho, 2009; Charles et al., 2002; Grinstein, 2008; Kasapoglu, 2011; Kowalkowski et al., 2017; Phillips et al., 2001).

\section{Metodología}

A partir de una investigación cualitativa, de corte analítico y descriptiva, se propone una revisión de la literatura, con el propósito de examinar los enfoques de la gestión administrativa y la orientación del mercado. Para ello, se obtuvieron publicaciones en bases de datos científicas, como Scopus y Web Of Science (WOS). El criterio de selección es fue el registro esté directamente relacionado con el tema tratado, además de ser publicaciones con mayor índice de citación en la literatura académica, esto se hizo con el fin de seleccionar aquellas publicaciones con mayor impacto académico. Posteriormente, se describieron y analizaron los resultados de la literatura, y a partir de ella se elaboraron los resultados y conclusiones basados en trabajos de investigación teóricos y empíricos.

\section{Resultados}

Revisando la literatura especializada en la creación de ventajas competitivas sostenibles para la orientación del mercado, podemos encontrar a grandes iconos de la mercadotecnia como lo son: Levitt 1960 y 1980; Kotler 1977 y 1984, Porter 1980 y 1985; Anderson 1982; Ohmae 1982; Peters y Waterman 1982; Day 1984; Aaker 1988, que la orientación al mercado es un concepto unidimensional, integrado por tres componentes de comportamiento $\mathrm{y}$ dos criterios de decisión. Como componentes de comportamiento se encuentran la necesidad de No.1 Orientarse al cliente, esto implica conseguir un gran bagaje de conocimientos en los compradores objetivos que faciliten y den posibilidad a la creación de un valor superior para ellos de forma continua, nos cita Torres (2015).

Es así que el objetivo es orientar al competidor, actual y potencial, identificando las fuerzas y debilidades a corto plazo y sus capacidades y estrategias a largo plazo. Seguidamente coordinar los recursos de la empresa con la creación de un valor superior para los clientes. Así mismo, tomar en cuenta los criterios de decisión con base a las necesidades de la empresa que externen una visión a largo plazo, tanto en la consecución de los beneficios como las propuestas de los componentes a definir, permitiendo la rentabilidad sobre el mercado.

En una segunda etapa, se contrasta la validez y fiabilidad del concepto elaborado, si bien queda reducido a los tres componentes de comportamiento. Esto es, la orientación al mercado, como forma de cultura organizativa requiere una orientación al cliente, al competidor y la coordinación inter-funcional, considerando que se debe asignar la misma importancia a cada uno de los tres componentes nos relata Torres (2015).

Ahora bien, los modelos antes descritos son, probablemente, los de mayor aceptación en el momento de explicar la orientación al mercado de una organización, sin embargo, no son los únicos desarrollados, revisando la literatura, podemos encontrar los trabajos de Kohli y Jaworski (1990) y Narver y Slater (1990) de los cuales tomaré solo uno para su desarrollo y comparación, así nos relata en la página web, Fichero virtual, (s.f.) La orientación al mercado:

Con base en los trabajos de Zaltman, LeMasters y Heffring (1982),Shapi-ro (1988) y Kohli y Jaworski (1990) conceptúan la orientación al mercado en torno a tres elementos; El primer asociado con la generación de inteligencia de mercado, esto implica la generación por parte de la organización de una inteligencia de mercado es un proceso que va más allá de solo recolección de datos y tratamiento de la información con base a las necesidades y preferencias de los consumidores; incluye, el estudio de los factores exógenos (competidores, tecnología, regulación gubernamental) que influyen en esas necesidades y preferencias actuales y que seguramente incidirán en las futuras.

En segundo lugar, su diseminación por el conjunto de departamentos, es decir, con la información disponible y convenientemente tratada, la organización debe proceder a su diseminación o separación hasta impregnar a todos y cada uno de los departamentos y a los individuos que los integran. Se trata así de abrir la posibilidad de que los departamentos trabajen en común para facilitar la concentración de las diferenciaciones empresariales. Un papel destacado en el desempeño de esta función es el que representa la comunicación horizontal o flujo lateral de información intra e interdepartamental -consideramos necesario y formalmente establecido el flujo 
vertical de información.

1 y tercero La respuesta de la organización nos remite al conjunto de acciones que tienen lugar a partir de la inteligencia generada y diseminada internamente sobre las necesidades y deseos de los consumidores, las estrategias de la competencia y los factores ambientales. Esencialmente, esta respuesta toma la forma de mercados seleccionados y de productos y servicios diseñados para satisfacer las necesidades actuales y futuras de tales mercados.

Kohli, Jaworski y Kumar (1993) han diseñado y contrastado una escala de 20 variables con objeto

\section{Conclusión}

La orientación de mercado dentro de la mercadotecnia, requiere de bastante y constante revisión en un entorno tan competitivo como el que vivimos día a día. En toda la literatura revisada con anterioridad me sorprendió lo amplio y basto que se vuelve a ser notar la necesidad y obligación de la implementación de la mercadotecnia como motor de arranque y avance dentro de las empresas. Pueden abundar teorías y conceptos, pero yo me sigo quedando con la filosofía hecha frases de medir la puesta en práctica por parte de las empresas de una orientación al mercado formulada en función de los componentes antes citados. La escala refleja la destacada atención que se presta a los análisis de las necesidades de los consumidores y competidores, pero no refleja la atención que se ha de dispensar al canal de marketing y a las capacidades internas. Esta formulación es más explícita sobre la necesidad de implantar en el seno de la organización una actitud de marketing, al incidir en la obligada respuesta de toda la organización como uno de los componentes clave de la orientación al mercado.

Según Denison y McDonald (1995): "El éxito en marketing no consiste solamente en identificar cuál es la estrategia adecuada, sino, además, en poder implantarla correctamente". Por otro lado, citando la misma conceptualización y filosofía de los autores antes mencionados, Lambin (1995) explica el hecho que: "Comprender el concepto marketing es una cosa, ponerlo en práctica es otra" siendo ésta en donde las empresas parecen haber hallado un mayor problema al limitarse a poner en práctica sus propios preceptos.

\section{Referencias}

Aaker, D. a. (2008). Marketing in a Silo World: THE NEW CMO CHALLENGE. California Management Review, 51(1), 144-156. https://doi.org/10.2307/41166473

Augusto, M., \& Coelho, F. (2009). Market orientation and new-to-the-world products: Exploring the moderating effects of innovativeness, competitive strength, and environmental forces. Industrial Marketing Management, 38(1), 94-108. https://doi.org/10.1016/j.indmarman.2007.09.007

Charles, H. N., Rajiv, K. S., \& Ajith., K. (2002). Market orientation and alternative strategic orientations: A longitudinal assessment of performance implications. Journal of Marketing, 66(October), 25-39. https://scholar.google.com/scholar?q=Noble,+Sinha,+\&+Kumar,+2002,+orientation $+\& b \operatorname{tnG}=\& h l=$ en\&as_sdt $=0,5 \# 9$

Chen, Y.-S., \& Chen, C.-C. (2009). A model of factors moderating the relationship between new product development and company performance. Social Behavior and Personality: An International Journal, 37(8), 1043-1049. https://doi.org/10.2224/sbp.2009.37.8.1043

David, F. R. (2019). Conceptos de Administración Estratégica.

Farzad; A., Nahavandi;, N., \& Caruana, A. (2008). The Effect of Internal Marketing on Organizational Commitment in Iranian Banks Atousa Farzad , 2 Nasim Nahavandi , 3 Albert Caruana Department of Industrial Engineering, Tarbiat Modares University, Tehran , Iran Department of Marketing, University of M. American Journal of Applied Sciences, 5(11), 1480-1486. 
Fraj, E., Matute, J., \& Melero, I. (2015). Environmental strategies and organizational competitiveness in the hotel industry: The role of learning and innovation as determinants of environmental success. Tourism Management, 46, 30-42. https://doi.org/10.1016/j.tourman.2014.05.009

Frías-Navarro \& Pascual. (2012). Exploratory Factor Analysis (Efa) in Consumer Behavior and Marketing Research. Suma Psicológica, 19(1), 47-58. https://doi.org/ISSN 0121-4381

Garcia, F., Jin, B. C., \& Salomon, R. (2013). Does inward foreign direct investment improve the innovative performance of local firms? Research Policy, 42(1), 231-244. https://doi.org/10.1016/j. respol.2012.06.005

Grinstein, A. (2008). The relationships between market orientation and alternative strategic orientations. 42(1), 115-134. https://doi.org/10.1108/03090560810840934

Guzmán Duque, A. P., \& Abreo Villamizar, C. A. (2017). Del comercio electrónico al comercio social: La innovación al alcance de las organizaciones. Estudio para el sector calzado Bucaramanga, Colombia. Contabilidad y Negocios, 12(24), 107-118. https://doi.org/10.18800/contabilidad.201702.006

Jiménez, P. (2010). La orientación al mercado, evolución y medición de un enfoque de gestión que trasciende al marketing perspectivas. Universidad Catolica Boliviana San Pablo. https://www.redalyc.org/pdf/4259/425942454004.pdf

Kasapoglu, E. (2011). Leadership Behaviors in Project Design Offices. Journal of Construction Engineering and Management, 137(5), 356-363. https://doi.org/10.1061/(ASCE)CO.19437862.0000308

Kashmiri, S., Nicol, C. D., \& Arora, S. (2017). Me, myself, and I: influence of CEO narcissism on firms' innovation strategy and the likelihood of product-harm crises. Journal of the Academy of Marketing Science, 45(5), 633-656. https://doi.org/10.1007/s11747-017-0535-8

Kotler, P., y Keller, K., (2016). Dirección de Marketing. Pearson Educación. Decimoquinta Edición.

Kowalkowski, C., Gebauer, H., \& Oliva, R. (2017). Service growth in product firms: Past, present, and future. Industrial Marketing Management, 60, 82-88. https://doi.org/10.1016/j.indmarman.2016.10.015

Larke, R., Kilgour, M., \& O’Connor, H. (2018). Build touchpoints and they will come: transitioning to omnichannel retailing. International Journal of Physical Distribution and Logistics Management, 48(4), 465-483. https://doi.org/10.1108/IJPDLM-09-2016-0276

Lázaro, J. (2016). La orientación al mercado y los resultados de la organización: influencia de los departamentos de marketing y ventas. Universitat Abat

Oliba CEU.

Longbottom, D., Mayer, R., \& Casey, J. (2000). Marketing, total quality management and benchmarking: Exploring the divide. Journal of Strategic Marketing, 8(4), 327-340. https://doi. org/10.1080/096525400446221

Lupoli Junior, J. G., \& Dos Santos, Y. M. (2019). Abordagens de marketing e orientação para mercado aplicadas às médias empresas brasileiras. Revista Ciências Administrativas, 25(3), 1-17. https://doi.org/10.5020/2318-0722.2019.8296

Madden, T. J., Fehle, F., \& Fournier, S. (2006). Brands matter: An empirical demonstration of the creation of shareholder value through branding. Journal of the Academy of Marketing Science, 34(2), 224-235. https://doi.org/10.1177/0092070305283356

Manes, J. M. (2014). Gestión estratégica para instituciones educativas. Ediciones Granica SA. 
Montoya, M. S. R. (2018). Modelos y estrategias de enseñanzas para ambientes innovadores. Editorial Digital del Tecnológico de Monterrey.

Morgan, N. A., \& Rego, L. L. (2009). Brand portfolio strategy and firm performance. Journal of Marketing, 73(1), 59-74. https://doi.org/10.1509/jmkg.73.1.59

Mueller, S. L., \& Thomas, A. S. (2001). Culture and entrepreneurial potential: A nine country study of locus of control and innovativeness. Journal of Business Venturing, 16(1), 51-75. https:// doi.org/10.1016/S0883-9026(99)00039-7

Nath, P., \& Mahajan, V. (2008). Chief Marketing Officers: A Study of Their Presence in Firms'Top Management Teams. Journal of Marketing, 72(1), 65-81. https://doi.org/10.1509/jmkg.72.1.65

Nowlin, E. L., Walker, D., \& Anaza, N. A. (2018). How does salesperson connectedness impact performance? It depends upon the level of internal volatility. Industrial Marketing Management, 68(January), 106-113. https://doi.org/10.1016/j.indmarman.2017.10.007

Park, C. W., MacInnis, D. J., Priester, J., Eisingerich, A. B., \& Iacobucci, D. (2010). Brand attachment and brand attitude strength. Journal of Marketing, 74(6), 1-17.

Phillips, P. A., Davies, F. M., \& Moutinho, L. (2001). The Interactive Effects of Strategic Marketing Planning and Performance: A Neural Network Analysis. Journal of Marketing Management, 17(1-2), 159-182. https://doi.org/10.1362/0267257012571438

Piercy, N. F., Harris, L. C., Peters, L. D., \& Lane, N. (1997). Marketing management, market strategy and strategic management: Domain realignment and redefinition. Journal of Strategic Marketing, 5(1), 50-62. https://doi.org/10.1080/096525497346866

Prieto, J., Pública, M. E. G., \& De, S. (2012). Gestión estratégica. Bogotá: Ecoe Ediciones.

Ramanathan, R., Ramanathan, U., \& Zhang, Y. (2016). Linking operations, marketing and environmental capabilities and diversification to hotel performance: A data envelopment analysis approach. International Journal of Production Economics, 176, 111-122. https://doi.org/10.1016/j.ijpe.2016.03.010

Rojas De Francisco, L., Bejarano Botero, L. M., \& Marín Valencia, C. F. (2016). Análisis de las estructuras de gestión del servicio en empresas del sector de servicios. AD-Minister, 29, 121146. https://doi.org/10.17230/ad-minister.29.6

Sainidis, E., Robson, A., \& Heron, G. (2019). Environmental turbulence and the role of business functions in the manufacturing strategy debate: The case of UK-based SMEs and the Great Recession. Journal of General Management, 44(4), 190-208. https://doi.org/10.1177/0306307019832498

Torres, R. (2015). La orientación al mercado y su medición. https://www.gestiopolis.com/la-orientacion-al-mercado-y-su-medicion/

Urde, M. (1994). Brands Today. Journal of Consumer Marketing, 11(3), 18-32. http://citeseerx.ist. psu.edu/viewdoc/download?doi=10.1.1.460.5774\&rep=rep1\&type=pdf

Wolters Kluwer, Guías Jurídicas, (2017). Orientación al mercado. 